\title{
Surface-treated 3D printed Ti-6Al-4V scaffolds with enhanced bone regeneration performance: an in vivo study
}

\author{
Guangdao Zhang ${ }^{1 \#}$, Pengyu Zhao ${ }^{2,3 \#}$, Lin Lin ${ }^{4}$, Limei Qin ${ }^{1}$, Zhiguang Huan ${ }^{2,3}$, Sander Leeflang ${ }^{5}$, Amir A. \\ Zadpoor $^{5}$, Jie Zhou ${ }^{5}$, Lin $\mathrm{Wu}^{1}$
}

${ }^{1}$ Department of Prosthodontics, School of Stomatology, China Medical University, Shenyang, China; ${ }^{2}$ State Key Laboratory of High Performance Ceramics and Superfine Microstructure, Shanghai Institute of Ceramics, Chinese Academy of Sciences, Shanghai, China; ${ }^{3}$ Center of Materials Science and Optoelectronics Engineering, University of Chinese Academy of Sciences, Beijing, China; ${ }^{4}$ The First People's Hospital of Shenyang, Shenyang, China; ${ }^{5}$ Department of Biomechanical Engineering, Delft University of Technology, The Netherlands

Contributions: (I) Conception and design: G Zhang, Z Huan, L Wu; (II) Administrative support: Z Huan, L Wu; (III) Provision of study materials: G Zhang, Pengyu Zhao, L Lin, J Zhou; (IV) Collection and assembly of data: G Zhang, L Lin, L Qin; (V) Data analysis and interpretation: G Zhang, P Zhao, Z Huan, S Leeflang, AA Zadpoor, J Zhou, L Wu; (VI) Manuscript writing: All authors; (VII) Final approval of manuscript: All authors.

"These authors contributed equally to this work.

Correspondence to: Lin Wu. Department of Prosthodontics, School of Stomatology, China Medical University, Shenyang, China.

Email: wulin13@163.com; Zhiguang Huan. State Key Laboratory of High Performance Ceramics and Superfine Microstructure, Shanghai Institute of Ceramics, Chinese Academy of Sciences, Shanghai, China. Email: huanzhiguang@mail.sic.ac.cn.

Background: Given their highly adjustable and predictable properties, three-dimensional(3D) printed geometrically ordered porous biomaterials offer unique opportunities as orthopedic implants. The performance of such biomaterials is, however, as much a result of the surface properties of the struts as it is of the $3 \mathrm{D}$ porous structure. In our previous study, we have investigated the in vitro performances of selective laser melted (SLM) Ti-6Al-4V scaffolds which are surface modified by the bioactive glass (BG) and mesoporous bioactive glass (MBG), respectively. The results demonstrated that such modification enhanced the attachment, proliferation, and differentiation of human bone marrow stromal cells (hBMSC). Here, we take the next step by assessing the therapeutic potential of $3 \mathrm{D}$ printed Ti-6Al-4V scaffolds with BG and MBG surface modifications for bone regeneration in a rabbit bone defect model.

Methods: 3D printed Ti-6Al-4V scaffolds with BG and MBG surface modifications were implanted into the femoral condyle of the rabbits, the Ti-6Al-4V scaffolds without surface modification were used as the control. At week 3, 6, and 9 after the implantation, micro-computed tomography (micro-CT) imaging, fluorescence double-labeling to determine the mineral apposition rate (MAR), and histological analysis of non-decalcified sections were performed.

Results: We found significantly higher volumes of regenerated bone, significantly higher values of the relevant bone morphometric parameters, clear signs of bone matrix apposition and maturation, and the evidence of progressed angiogenesis and blood vessel formation in the groups where the bioactive glass was added as a coating, particularly the MGB group.

Conclusions: The MBG coating resulted in enhanced osteoconduction and vascularization in bone defect healing, which was attributed to the release of silicon and calcium ions and the presence of a nanomesoporous structure on the surface of the MBG specimens.

Keywords: Bone regeneration; 3D printing; bioactive glass; mesoporous bioactive glass; in vivo

Submitted May 09, 2020. Accepted for publication Sep 25, 2020.

doi: 10.21037/atm-20-3829

View this article at: http://dx.doi.org/10.21037/atm-20-3829 


\section{Introduction}

The full regeneration of the bone defects caused by trauma, infections, tumors, or the genetic disorders resulting in developmental skeletal anomalies, is a major clinical challenge $(1,2)$. Bone grafting is one of the most commonly used methods to treat bone defects. Autologous bone, or autograft, is considered to be the gold standard in clinical practice and the most effective method for bone regeneration $(3,4)$. However, autografting presents several disadvantages, with limited bone supply and donor site morbidity being the major drawbacks, especially if there is a massive bone loss due to trauma or disease (3,5-7). To overcome the limitations of the current treatment options, extensive research in the field of bone tissue engineering (BTE) has been directed towards creating novel alternatives to traditional bone grafts $(8,9)$. Threedimensional (3D) porous scaffolds prepared by using various fabrication methods and based on a wide range of biomaterials have been utilized to facilitate and guide bone regeneration (10-12). An ideal porous scaffold should be biocompatible and non-toxic, ensure normal cell adhesion, and promote cell migration and differentiation $(13,14)$. Besides, the scaffold structure should be similar to the natural bone tissue and have interconnected pores to facilitate the construction of a local vascular system during bone regeneration $(15,16)$. Furthermore, for a scaffold to be used in a load-bearing area, it must possess suitable mechanical properties to bear physiological loads on the one hand and to minimize the risk of stress shielding on the other $(17,18)$.

Titanium alloys have been widely used in orthopedic and dental treatments due to their excellent biocompatibility and favorable mechanical properties. In recent years, with the ever-increasing application of additive manufacturing (AM, or 3D printing) techniques in the medicine (19-22), it has become feasible to process a chosen titanium alloy into porous scaffolds with high structural complexity, patient-specific geometry, and tunable mechanical properties and permeability as a bone replacement material $(23,24)$. However, due to the inherent bioinertness of titanium, the performance of a titanium-based biomaterial in osteoconduction and osteoinduction does not meet the requirements of an ideal scaffold for bone tissue regeneration, as mentioned earlier, which limits the applications of titanium alloy scaffolds in orthopedic treatments $(8,25,26)$.

The bone-bonding properties of bioactive glass (BG) were reported over 40 years ago (27). Since then, BG has been extensively used in a variety of biomedical applications including the repair of (mostly non-load-bearing) bony defects. In recent years, BG has exhibited great potential for bone tissue regeneration due to its excellent osteoconductive and osteostimulatory properties (28-32). Through the combination of the chemical sol-gel of the multi-component $\mathrm{SiO}_{2}-\mathrm{CaO}-\mathrm{P}_{2} \mathrm{O}_{5}$ system of $\mathrm{BG}$ with the supramolecular chemistry using surfactants as structuredirecting agents, mesoporous bioactive glass (MBG) with a porous nanostructure, large specific surface area, and very high bioactivity can be synthesized (33,34). MBG has been applied to modify the surface of $\beta$-tricalcium phosphate $(\beta$-TCP) scaffolds (35). Due to the release of Si ions from MBG and the mesoporous structure of MBG on the $\beta$-TCP strut surface, surface-modified $\beta$-TCP scaffolds show significantly improved apatite mineralization, osteogenic and angiogenic activities, and enhanced bone regeneration performance as compared to bare $\beta$-TCP scaffolds (35). In a previous study (23), BG and MBG coatings were applied onto the strut surfaces of selective laser melted (SLM) Ti$6 \mathrm{Al}-4 \mathrm{~V}$ scaffolds. After spin-coating and heat treatment to remove the organic compounds used in the preparation of the coating, the characteristic mesoporous structure and chemical composition of MBG were retained. The in vitro study confirmed great improvements in the attachment, proliferation, and differentiation of human bone marrow stromal cells (hBMSCs) on the MBG-coated Ti-6Al$4 \mathrm{~V}$ scaffolds (23). Here, we utilize the rabbit bone defect model to further evaluate the bone tissue regeneration performance of BG and MBG modified 3D printed Ti-6Al$4 \mathrm{~V}$ scaffolds.

We present the following article in accordance with the ARRIVE reporting checklist (available at http://dx.doi. org/10.21037/atm-20-3829).

\section{Methods}

Preparation of the BG and MBG coated Ti-6Al-4Vporous scaffolds

Cylindrical porous titanium specimens $\left(\Phi 5 \times 10 \mathrm{~mm}^{2}\right)$ were additively manufactured using an SLM machine (Realizer, SLM-125, Borchen, Germany) equipped with a YLM400-AC ytterbium fiber laser (IPG Photonics Corporation, Oxford, MA, USA) under inert argon atmosphere (argon) with an oxygen content of less than $0.2 \%$. A medical-grade Ti-6Al-4V powder (grade 23) with a median particle size of 
Table 1 The chemical composition of the Ti-6Al-4V powder (by weight percent)

\begin{tabular}{lccccccccc}
\hline Element & $\mathrm{C}$ & $\mathrm{O}$ & $\mathrm{N}$ & $\mathrm{H}$ & $\mathrm{Fe}$ & $\mathrm{Al}$ & $\mathrm{V}$ & $\mathrm{Ti}$ \\
\hline Content (wt.\%) & 0.02 & 0.10 & 0.02 & 0.0017 & 0.19 & 6.4 & 4.0 & Balance \\
\hline
\end{tabular}

$36 \mu \mathrm{m}$ (Advanced Powders and Coating, Boisbriand, QC, Canada) was used in scaffold manufacturing. The chemical composition of the powder is presented in Table 1. The scaffolds had a lattice structure of repeated diamond unit cells with a strut thickness of $300 \mu \mathrm{m}$, corresponding to a porosity value of $68 \%$ (design value). The as-built samples were cleaned by wiping and ultrasonication in acetone to remove powder particles loosely attached to the surfaces of the samples. No further post-SLM processing was performed.

Then, a $\mathrm{SiO}_{2}$ interlayer was applied to the sample surface before coating to avoid interactions between the Ti-6Al-4V substrate and MBG coating, which might cause changes in the chemical composition and mesoporous structure of MBG. Tetraethyl orthosilicate (TEOS) (20.1 g) was hydrolyzed in $180 \mathrm{~g}$ ethanol solution, using $0.5 \mathrm{M}$ $\mathrm{HCl}$ as the catalyzer. An MBG precursor solution (molar composition: $80 \mathrm{Si}-15 \mathrm{Ca}-5 \mathrm{P}$ ) was prepared in the same way as reported in the previous study (23). Briefly, $12 \mathrm{~g}$ of nonionic block copolymer $\mathrm{EO}_{20} \mathrm{PO}_{70} \mathrm{EO}_{20}$ (P123, SigmaAldrich, St. Louis, MO, USA) was dissolved in $180 \mathrm{~g}$ ethanol and then stirred to clarification. Then, $20.1 \mathrm{~g}$ of TEOS, $4.2 \mathrm{~g} \mathrm{Ca}\left(\mathrm{NO}_{3}\right)_{2} \cdot 4 \mathrm{H}_{2} \mathrm{O}, 2.19 \mathrm{~g}$ triethyl phosphate (TEP, $99.8 \%$ ), and $3 \mathrm{~g}$ of $0.5 \mathrm{M} \mathrm{HCl}$ were added. The solution was stirred for $24 \mathrm{~h}$. TEOS, $\mathrm{Ca}\left(\mathrm{NO}_{3}\right)_{2} \cdot 4 \mathrm{H}_{2} \mathrm{O}$, TEP, and $\mathrm{HCl}$ of the analytical reagent (AR) grade were purchased from Sinopharm Chemical Reagent, Shanghai, China. The obtained $\mathrm{SiO}_{2}$ precursor solution was used for coating on the surfaces of the samples by using the spin coating method four times successively, at a rotational speed of $500 \mathrm{rpm}$ during the first $10 \mathrm{~s}$ and 2,000 rpm during the next $20 \mathrm{~s}$. Between the two coating runs, the specimens were placed in a fume hood for $8 \mathrm{~h}$ to allow the organic compounds to evaporate. Then, the MBG precursor solution was used for coating on the scaffold surfaces in the same way. The evaporation-induced self-assembly (EISA) method was used to obtain the dried gel. Finally, the coated scaffold samples were heated at a rate of $1{ }^{\circ} \mathrm{C} / \mathrm{min}$ to $650{ }^{\circ} \mathrm{C}$ and held at $650^{\circ} \mathrm{C}$ for $5 \mathrm{~h}$ to extirpate the organic compounds and form the mesoporous structure. Also, bioactive glass (BG) coating without mesopores was applied to the strut surfaces of the Ti-6Al-4V scaffolds for comparison purposes. No P123 was added to the BG precursor during preparation. $\mathrm{The}^{\mathrm{SiO}_{2}}$ precursor was the same as that applied to the MBG-coated scaffolds (Figure 1). The samples were ultrasonically cleaned and sterilized at $134{ }^{\circ} \mathrm{C} / 0.21 \mathrm{MPa}$ in an autoclave before implantation.

\section{Animals and porous scaffolds implantation}

The general guidelines on the use of animals in research have complied with all these experiments. The study was approved by the Animal Ethics Committee of China Medical University (No. K2016014). Eighteen mature male New Zealand white rabbits (authorization number: SCXK LU 2015 0001), provided by the Xilingjiao Breeding Center of China, Jinan, China with an average body weight of $2.5 \mathrm{~kg}(\mathrm{SD}=0.3 \mathrm{~kg})$ were used for the in vivo study. The rabbits were acclimated for 2 weeks before the study; they were housed in climate-controlled quarters with a $12 \mathrm{~h} / 12 \mathrm{~h} \mathrm{light/dark}$ cycle, with free access to food and water. These rabbits were randomly divided into three groups, namely Ti, Ti-BG, and Ti-MBG ( $\mathrm{n}=6)$. General anesthesia was induced by intramuscular injection with $3 \%$ pentobarbital sodium (1 mg/kg of body weight, Sinopharm Chemical Reagent, Shanghai, China). Local anesthesia was induced by subcutaneous injection with $1 \%$ lidocaine (0.5 mL, Sinopharm Chemical Reagent, Shanghai, China). Under sterile conditions, a 2 -cm skin incision was made on the distal femoral condyle with split periosteum. An implantation hole with a diameter of $5.5 \mathrm{~mm}$ and a depth of $10 \mathrm{~mm}$ was drilled using a W\&H implanted motor SI923 (W\&H Group, Bürmoos, Austria) and a trephine drill $(\Phi=5.5 \mathrm{~mm})$ on both femoral condyles (Figure $2 A)$. During drilling, the site was irrigated using a $0.9 \% 4{ }^{\circ} \mathrm{C}$ sterile saline solution to minimize friction and thermal necrosis. A cylindrical porous scaffold (with a diameter of $5 \mathrm{~mm}$ and a length of $10 \mathrm{~mm}$ ) was then press-fitted into the defect (Figure 2B). The subcutaneous tissue and skin were sutured in different layers.

Both sides of the femoral condyle were operated on, and the same kinds of porous scaffolds were implanted into the right and left defects in every group. After the surgery, the rabbits were individually caged and fed. The animals 

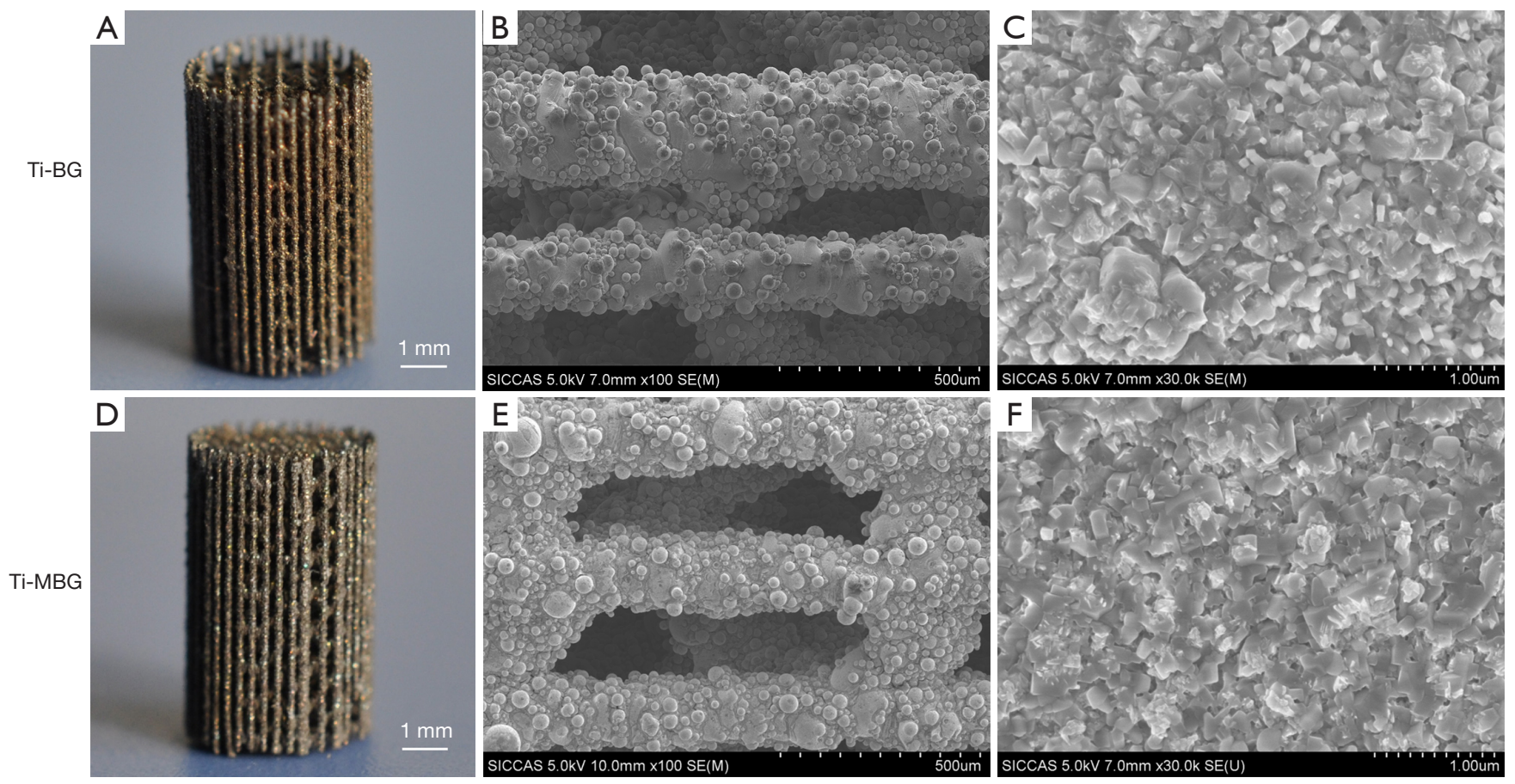

Figure 1 The characteristics of the BG- and MBG-coated Ti-6Al-4Vporous scaffolds. Overview and SEM images of BG-coated Ti-6Al4Vporous scaffolds (upper row); Overview and SEM images of MBG-coated Ti-6Al-4V porous scaffolds (lower row). BG, bioactive glass; MBG, mesoporous bioactive glass; SEM, scanning electron microscope.

were subcutaneously injected with $5 \mathrm{mg} / \mathrm{kg}$ calcein and $90 \mathrm{mg} / \mathrm{kg}$ xylenol orange (Sinopharm Chemical Reagent, Shanghai, China), respectively, at 2 weeks and 1 week before being sacrificed for fluorescence double-labeling. At week 3, 6, and 9, two rabbits in every group were sacrificed successively. Both the right and left femur condyles were retrieved and preserved in $4 \%$ paraformaldehyde at $4{ }^{\circ} \mathrm{C}$ for subsequent analyses.

\section{Micro-CT imaging}

After being fixed in paraformaldehyde for 1 week, the femoral condyles were scanned using micro-computed tomography (micro-CT) (YXLON Cheetah, Hamburg, Germany). Four samples were prepared for each group at week 3, 6 and 9. The tube voltage, tube current, and voxel sizes were $90 \mathrm{kV}, 50 \mu \mathrm{A}$, and $20.0 \mu \mathrm{m} \times 20.0 \mu \mathrm{m} \times 20.0 \mu \mathrm{m}$, respectively. An image analysis software suite (VGStudio Max 2.2.2, Volume Graphics, Heidelberg, Germany) was used to analyze and visualize the micro-CT data. To assess the peripheral and central bone formation, respectively, around and inside the porous scaffolds, a cylindrical volume $(5.5 \mathrm{~mm}$ in diameter and $10 \mathrm{~mm}$ in height) with the porous scaffold in the center was selected as the region of interest (ROI), and 3D reconstruction was performed (Figure 3).

In this ROI, the bone volume/total volume (BV/TV) and structural bone parameters, including trabecular thickness (Tb.Th), trabecular number (Tb.N), and trabecular separation (Tb.Sp), were determined.

\section{Preparation of hard tissue sections}

Following micro-CT imaging, the samples were processed for microscopy. They were rinsed with running water for $24 \mathrm{~h}$, dehydrated in a series of ascending ethanol concentrations $(50 \%, 75 \%, 95 \%$, and $100 \%)$, and then embedded in methyl methacrylate (PMMA, Heraeus Kulzer, Hanau, Germany). The samples were trimmed and mounted for sectioning. Several sections (thickness $=100-120 \mu \mathrm{m}$ ) were cut in the central part of the samples using a Leica-SP 1600 diamond-saw microtome (Leica Instruments, Nußloch, Germany) perpendicularly to the long axis of the porous scaffold. The sections were then reduced to a final thickness of approximately $50 \mu \mathrm{m}$ by using silicon carbide grinding cloths successively, followed by polishing with an alumina suspension (36). Two middle sections of each sample were 

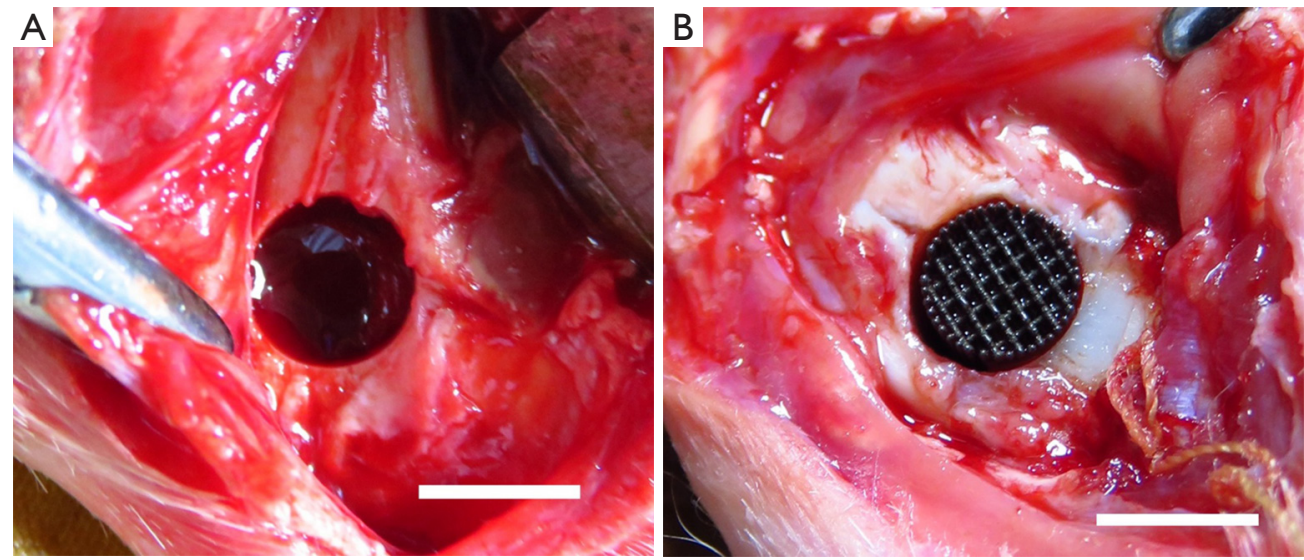

Figure 2 Images of porous scaffolds implantation. The holes drilled in the femoral condyles of rabbits with a diameter of $5.5 \mathrm{~mm}$ and a depth of $10 \mathrm{~mm}$ (A). The holes were subsequently filled with porous scaffolds with a diameter of $5 \mathrm{~mm}$ and a length of $10 \mathrm{~mm}$ (scale bars: $5 \mathrm{~mm})(\mathrm{B})$.

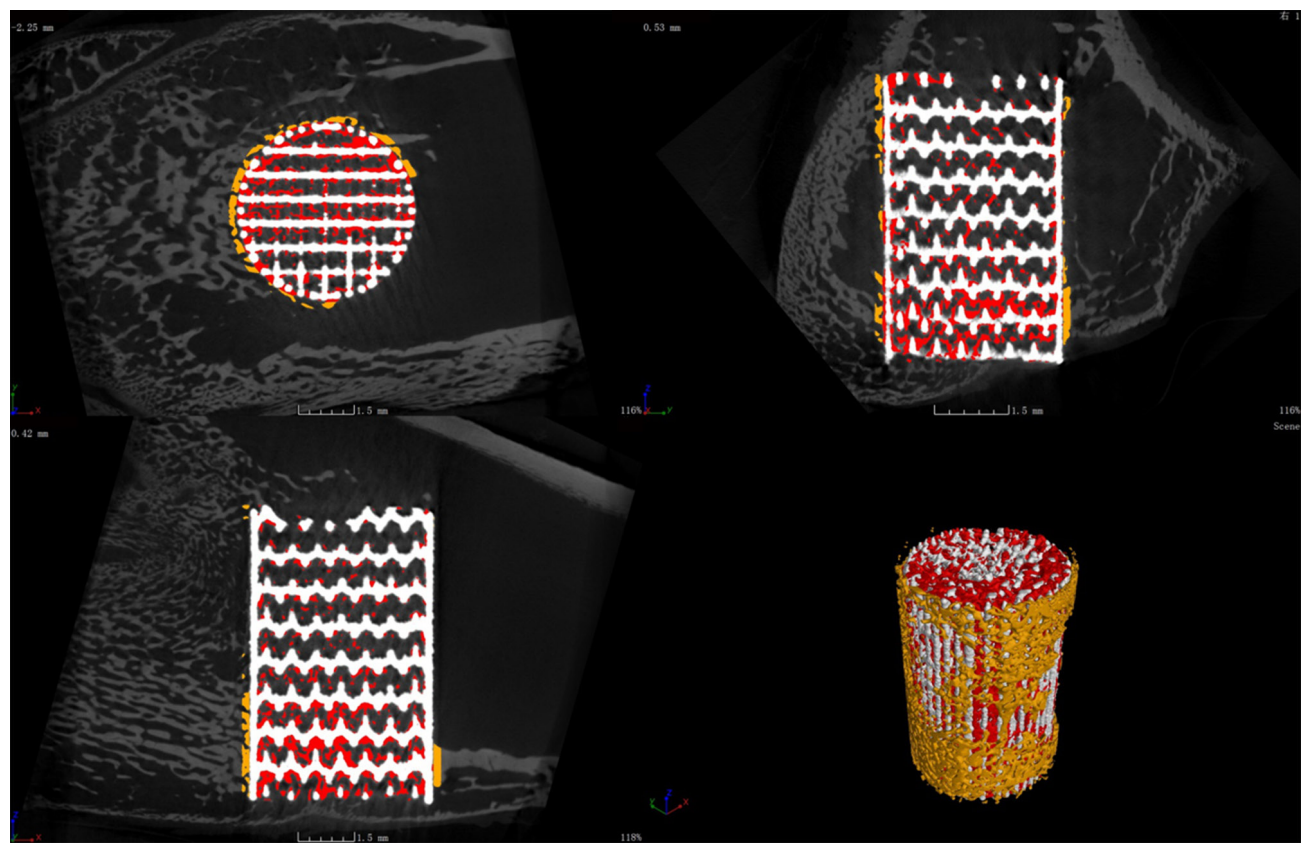

Figure 3 Micro-CT images and 3D reconstruction. A cylindrical volume (with a diameter of $5.5 \mathrm{~mm}$ and a height of $10 \mathrm{~mm}$ ) with the titanium alloy porous scaffold in its center was selected as the region of interest (ROI). The volumes of the de novo bone formed in the central part and around the periphery of the porous scaffold are shown in yellow and red, respectively. The porous titanium alloy scaffold is depicted in white. Micro-CT, micro-computed tomography; 3D, three-dimensional.

selected for fluorescence microscopy and histological methylene blue acid fuchsin (BM-AF) staining.

\section{Fluorescence microscopy}

The fluorescent double labels (calcein and xylenol orange) of the central newly-formed bone in the porous scaffolds were detected on non-decalcified sections by a fluorescence microscope (Olympus BX51 + DP71, Japan). Four sections from each group of the scaffolds at each of the time points were examined. Each section with a fluorescent dye was analyzed by using the ImageJ software (National 
Institutes of Health, USA). As the different fluorescence emission spectra in the image represent the apposition of de novo bone at different time points, the area of interest was selected to quantify the fluorescence intensity of the labeled area, which reflected the formation of de novo bone during the corresponding period. One parallel fluorescence double labeling region was selected in each section, and the distance between the red and green bands was measured at both ends of the region. Then, the mineral apposition rate (MAR = the distance of fluorescence double labeling/time) was determined. MAR represented the rate of de novo bone mineralization in the three different groups of the scaffolds after implantation.

\section{Statistical analyses}

The experimental results are presented as mean $\pm \mathrm{SD}$ (standard deviation). Statistical analyses were performed with the SPSS Statistics 21.0 software (IBM, Armonk, USA). Significant differences between the groups in the abovementioned measurements were compared using a one-way analysis of variation combined with the Student-NewmanKeuls (SNK) post hoc test. For all the tests, results with a $\mathrm{P}$ value $<0.05$ were considered to be statistically significant.

\section{Results}

\section{Micro-CT images and quantitative analysis}

Quantitative measurements of the de novo bone formed in the central and peripheral regions using reconstructed micro-CT images (Figure 4) showed that, regardless of the type of scaffold, BV/TV increased with implantation time (Figure 5). However, there were significant differences between the different experimental groups: the BV/TV values corresponding with the Ti-BG and Ti-MBG groups were significantly higher than that of the Ti group for all the considered time points (Figure 5). At week 6, the BV/ TV value of the Ti-MBG group was $7.24 \%$ higher than that of the Ti-BG group $(\mathrm{P}<0.05, \mathrm{n}=4)$. A similar trend was observed for the trabecular thickness (Tb.Th): for all the experimental groups, the Tb.Th value corresponding with de novo bone increased with implantation time (Figure 5). At week 3, the Tb.Th value of the Ti-MBG group was significantly higher than the values of both $\mathrm{Ti}$ and Ti-BG groups. At week 6, the Ti-MBG group had a higher Tb.Th value than that of the Ti-BG group, while the Ti-BG group had a higher Tb.Th value than that of the
Ti group. At week 9, the Tb.Th values of the Ti-MBG and Ti-BG groups were both significantly higher than the value of the Ti group.

As for the trabecular number (Tb.N), the value corresponding with the Ti-MBG group was significantly higher than that of the Ti-BG group at week 6 , while the Ti-BG group had a higher Tb.N value than that of the Ti group at the same time point (Figure 5). At week 9, however, the increase in the Tb.N value of the Ti-MBG group slowed down, and there was no statistically significant difference between the Ti-BG and Ti-MBG groups. Both values were still higher than the $\mathrm{Tb} . \mathrm{N}$ value of the $\mathrm{Ti}$ group.

An analysis of the trabecular spacing (Tb.Sp) showed that the Tb.Sp values gradually decreasing with implantation time and that Tb.Sp was inversely correlated with Tb.N (the Pearson's correlation coefficient: Ti $=-0.9968$, Ti$\mathrm{BG}=-0.8726$, Ti-MBG $=-0.9374)$. The obtained results confirmed the regularity in the formation of de novo bone and the maturation of the bone formed within the porous scaffolds after implantation.

The same bone morphological parameters were also calculated for the peripherally formed de novo bone (Figure 6). At all considered time points, the Ti-MBG group had significantly higher values of BV/TV and Tb.Th than the Ti group. Interestingly, the Tb.N values of the Ti-BG and Ti groups showed a decreasing trend from week 6 to 9 , while the Tb.N value of the Ti-MBG group was still on the rise. The Tb.Sp value of the Ti-MBG group decreased with implantation time, while the Tb.Sp values measured for the bare Ti scaffolds fluctuated throughout the 9-week followup period.

\section{Fluorescence double labeling for MAR}

Figure 7 shows the representative images of the fluorescent double-labeled de novo bone of the Ti, Ti-BG, and TiMBG groups. The calcein and xylenol orange fluorescent labels represented the new mineralized bone over 2 weeks and 1 week before the euthanasia of animals, respectively. It can be observed that the green fluorescent labels are closer to the site of the implanted material, indicating that the de novo bone covered the surface of the scaffold first and then occupied the scaffold pores.

The MAR values of the Ti-MBG $(2.248 \pm 0.374)$ and TiBG groups $(2.175 \pm 0.210)$ were significantly higher than the value of the Ti group $(1.720 \pm 0.244)$ at week $3(\mathrm{P}<0.05, \mathrm{n}=4)$ (Figure 8). This confirmed that the application of the MBG 
3 Weeks
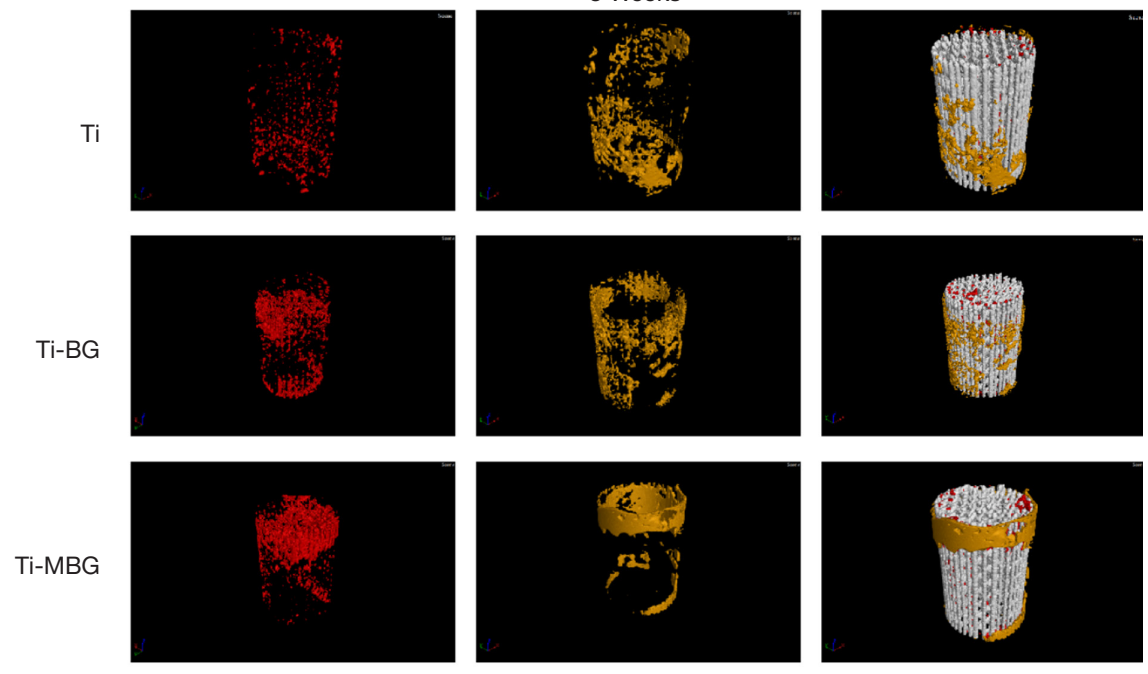

6 Weeks
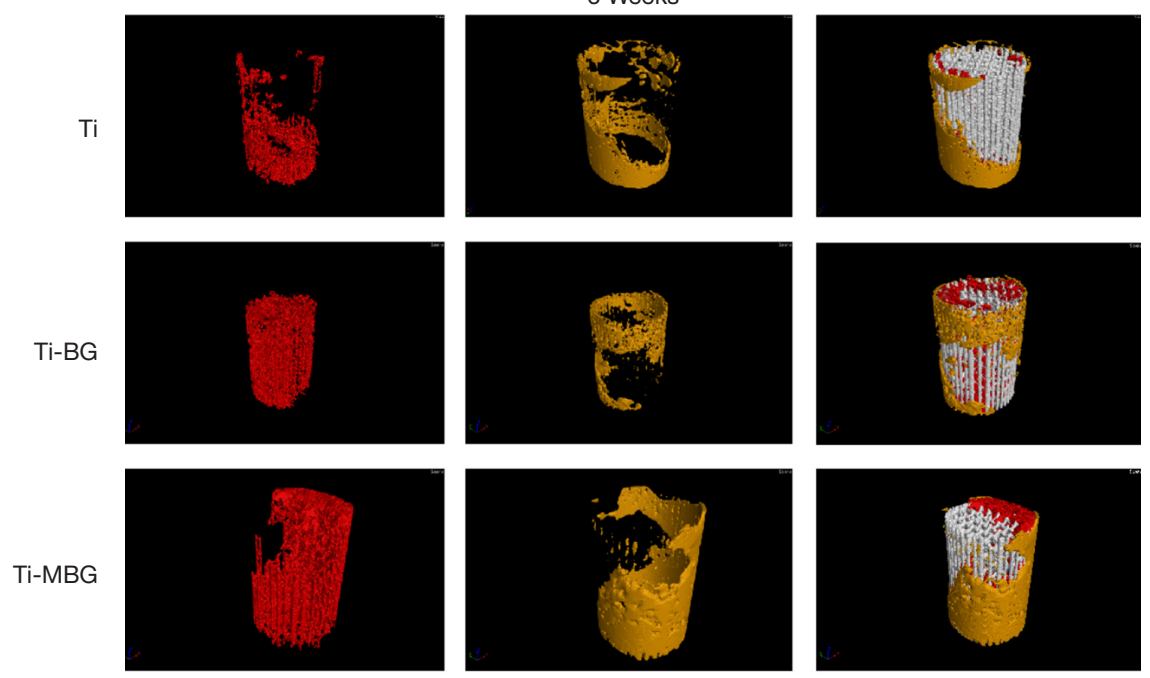

9 Weeks
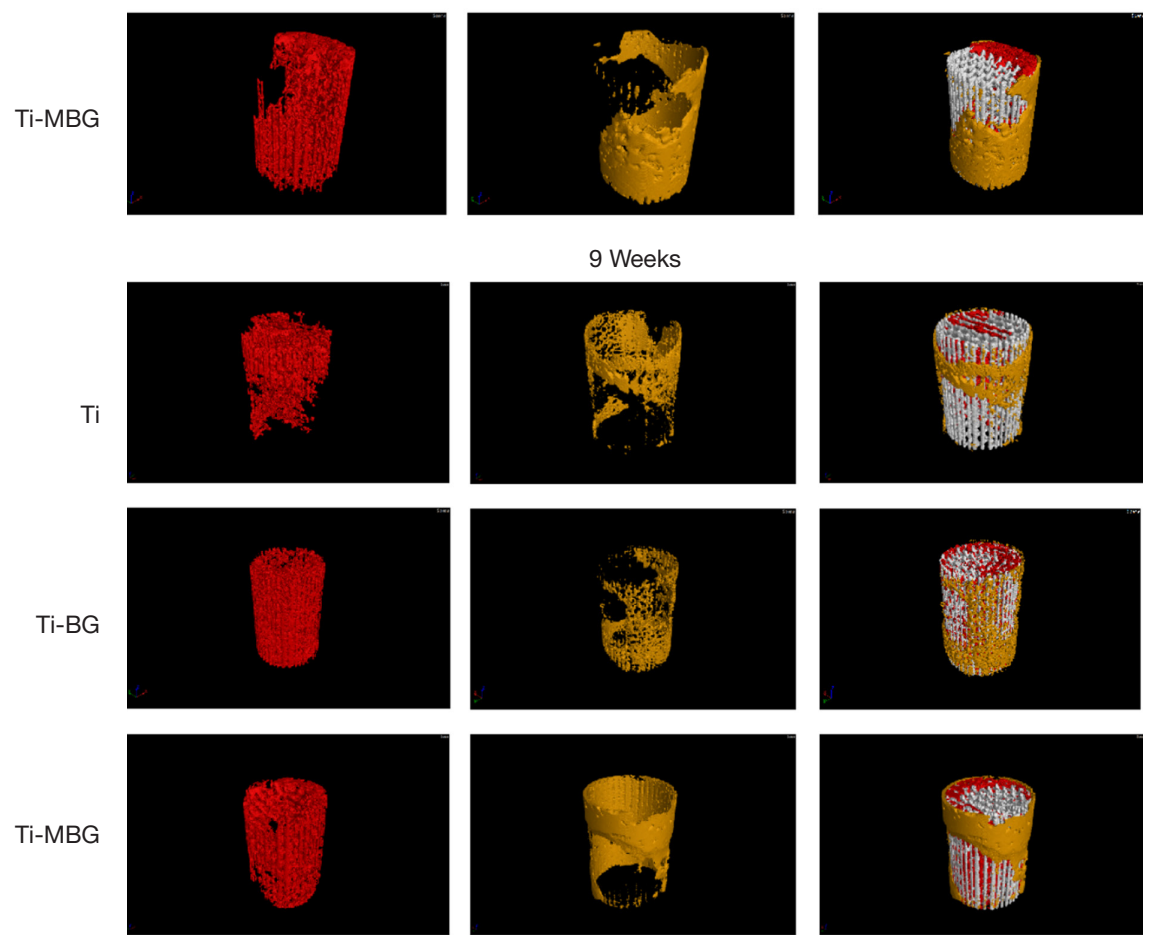

Figure 4 3D reconstructions of the de novo bone at week 3, 6, and 9. The same color code as in Figure 3 is used here. 

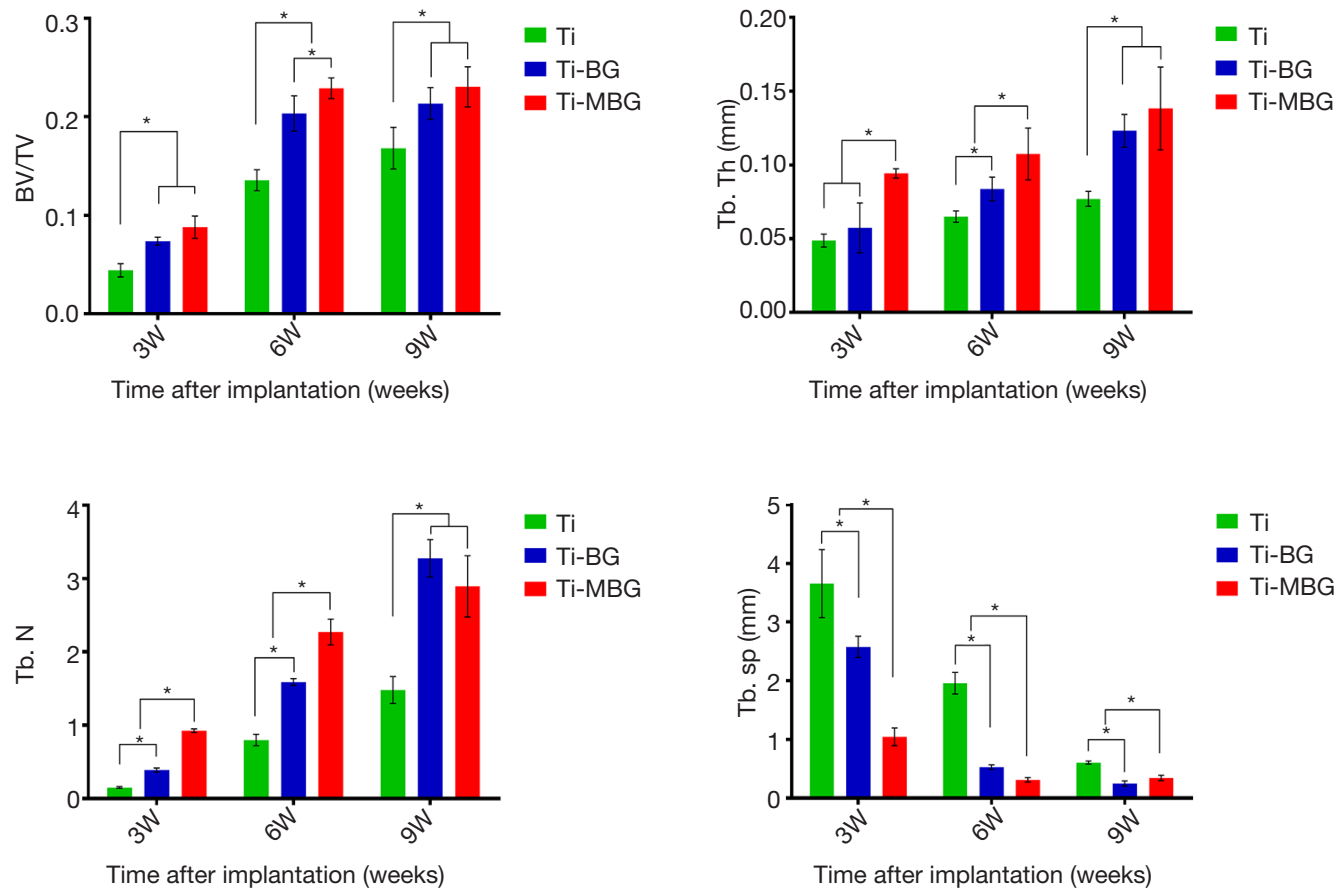

Figure 5 The different parameters describing the quantity and quality of centrally formed de novo bone in all experimental groups at week 3 , 6, and 9. BV/TV, bone volume/total volume; Tb.Th, trabecular thickness; Tb.N, trabecular number; Tb.Sp, trabecular separation ( ${ }^{*}$ indicates $\mathrm{P}<0.05)$.

and BG coatings could, indeed, improve the rate of the mineralization of de novo bone inside the porous scaffolds at the early stages. Through week 6 , the MAR values of the Ti-MBG and Ti-BG groups continued to increase to $3.230 \pm 0.232$ and $2.758 \pm 0.389$, respectively. These values were significantly higher than the value of the Ti group $(2.368 \pm 0.141)(\mathrm{P}<0.05, \mathrm{n}=4)$. At week 9, the MAR value of the Ti Group was found to have increased to $2.730 \pm 0.145$. In contrast, the MAR values of the Ti-MBG and TiBG groups decreased to $2.079 \pm 0.347$ and $2.103 \pm 0.226$, respectively. These values were significantly different from the value of the Ti group $(\mathrm{P}<0.05, \mathrm{n}=4)$.

\section{Histological observations of non-decalcified sections by $B M-A F$ staining}

At week 3, a small amount of woven bone (WB) had formed in the porous scaffolds of the Ti group, while massive $\mathrm{WB}$ formation was observed for the Ti-MBG and Ti-BG groups (Figure 9). At week 6, the WB in the Ti group had matured further and the trabeculae widened, but mature lamellar bone (LB) was rarely visible. In the Ti-MBG and Ti-BG groups, trabeculae had widened and occupied most of the scaffold pores. The mature LB appeared in the porous scaffolds and vascular distribution could be seen in the de novo bone. At week 9, the trabeculae observed in the $\mathrm{Ti}$ group had widened and some mature LB had appeared, but not all the scaffold space was occupied. A few blood vessels were also observed in the newly formed bone tissue. In the Ti-MBG and Ti-BG groups, the bone tissue matured further and the degree of vascularization was higher than the Ti group. The Ti-MBG group tended to present a larger number of blood vessels than the Ti-BG group. Most of the scaffold space was occupied by newly formed bone. Many typical Haversian systems could be observed. These results indicated that the de novo bone formed in the Ti-MBG and Ti-BG groups had entered the mature bone stage.

\section{Discussion}

Biocompatible metals are often considered to be suitable biomaterials for orthopedic implants and bone tissue engineering scaffolds, due to their high mechanical strength and ductility as compared to polymeric and ceramic biomaterials $(5,8,25)$. The main issues associated with 

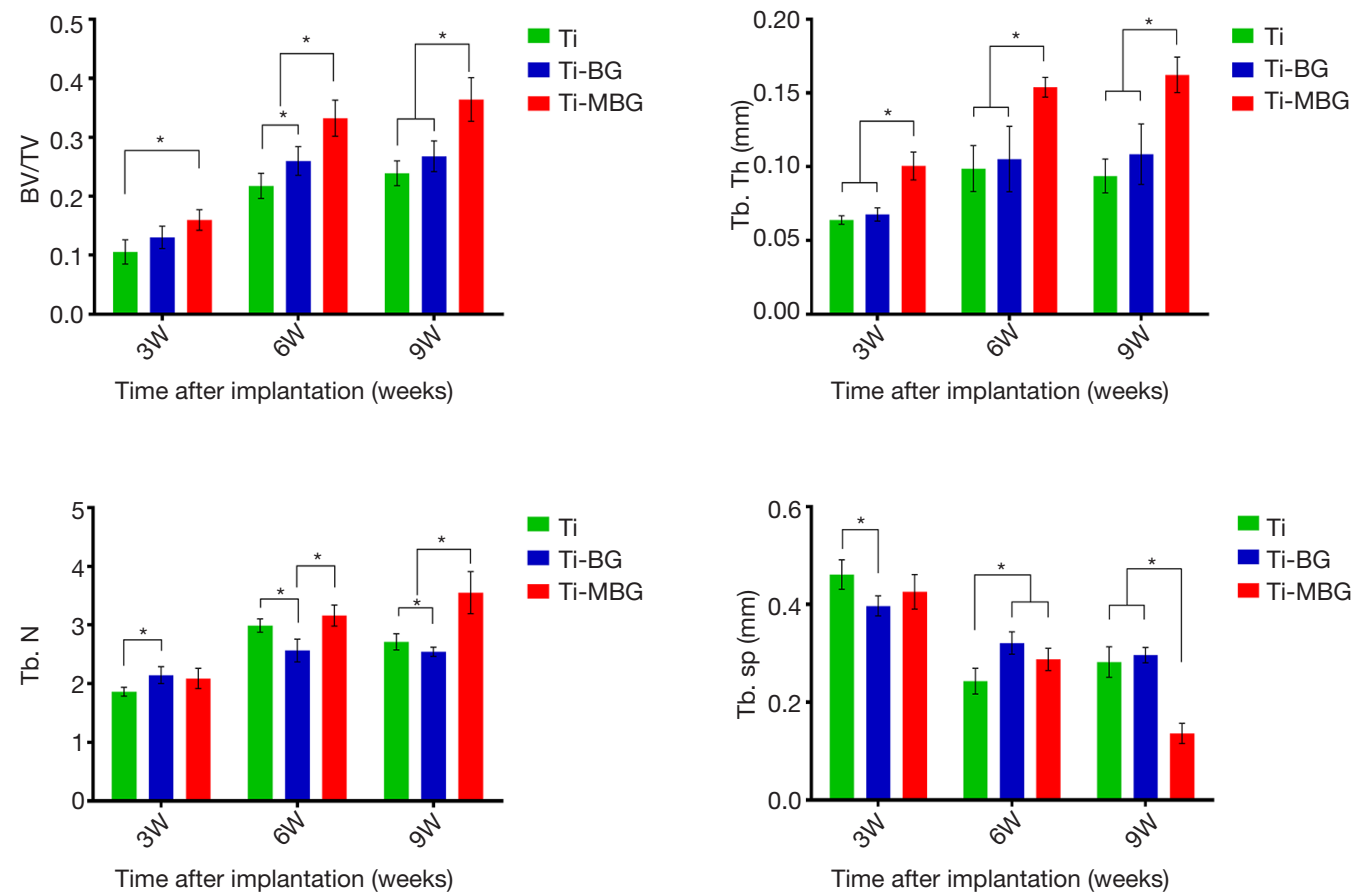

Figure 6 The different parameters describing the quantity and quality of peripherally formed de novo bone in all experimental groups at week 3, 6, and 9. BV/TV, bone volume/total volume; Tb.Th, trabecular thickness; Tb.N, trabecular number; Tb.Sp, trabecular separation (*indicates $\mathrm{P}<0.05$ ).

metallic implants are the bone resorption and loosening caused by stress shielding, weak interfacial bonding between the implant and bone, and the lack of biological anchorage for the growing tissue (37). To resolve these issues, new types of porous metallic biomaterials whose architecture and mechanical properties resemble those of bone have been developed $(25,38)$. Such scaffolds are typically designed and fabricated to be highly porous and have pores of suitable sizes at all locations throughout the scaffolds to provide an optimum environment for the formation of the bony matrix $(39,40)$. SLM, as one of the AM technologies, has been used in the fabrication of complex and personalized metallic scaffolds in the medical field. Using computer-aided design and SLM, the macroscopic shape, lattice structure, strut thickness, and porosity of the scaffolds can be accurately controlled. In this study, we successfully fabricated cylindrical porous Ti-6Al-4V scaffolds with a diameter of $5 \mathrm{~mm}$ and a height of $10 \mathrm{~mm}$ by using the SLM technology. The pore sizes of the porous implants were between 150 and $500 \mu \mathrm{m}$ with total porosity of $68 \%$ as the design value. As shown in BM-AF staining (Figure 9), WB was observed in the bare-metal Ti-6Al-4V scaffolds at week 3 after implantation. At week 9, trabeculae widened and some mature LB appeared, while nearly $50 \%$ of the available pore space was occupied by de novo bone. Besides, a few blood vessels were observed in the newly formed bone tissue. These results indicate that $3 \mathrm{D}$ printed $\mathrm{Ti}-6 \mathrm{Al}-4 \mathrm{~V}$ scaffolds with an interconnected pore structure enable blood vessel formation and bone ingrowth. Therefore, they can be used for the treatment of femoral condyle defects. However, the fact that only $50 \%$ of the pore space was occupied by de novo bone at week 9 suggests that the osteoinductive capability of the bare-metal Ti-6Al-4V scaffolds is not high enough to enable full regeneration of bone in the scaffolds.

Surface modification with a bioactive coating on titanium alloys has been proven to be one of the most effective strategies to improve the osseointegration capability and biocompatibility of titanium-based implants $(41,42)$. BG, which is biodegradable in vivo and releases silicon and calcium ions during its degradation, has received increasing attention for many years by the researchers active in the field of advanced bioceramics. Due to its well-ordered mesoporous structure as compared to the conventional BG without a mesoporous structure, MBG significantly improves its specific surface area, pore volume, and apatite formation ability (35). Despite its superior bioactivity 

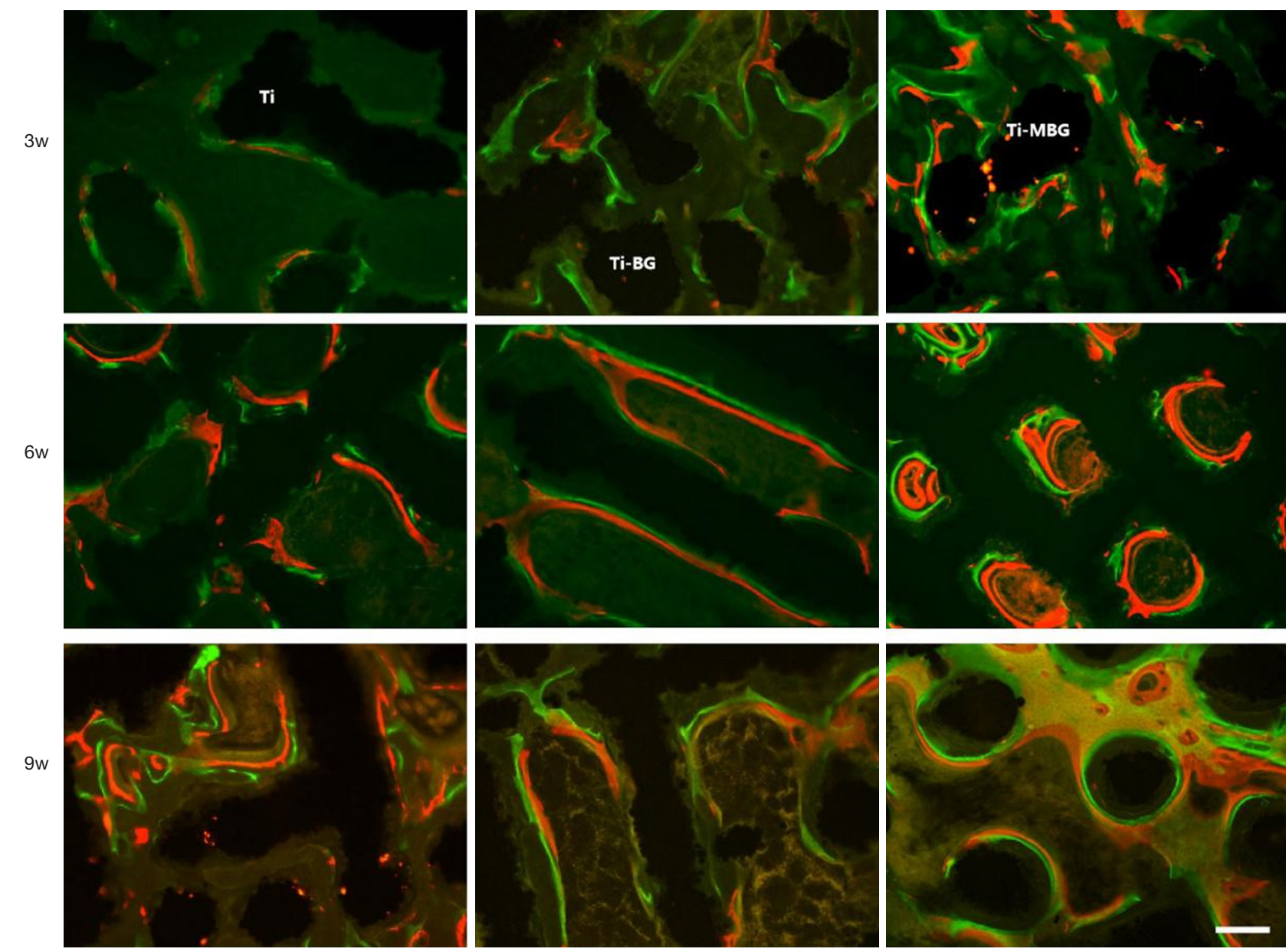

Ti-BG

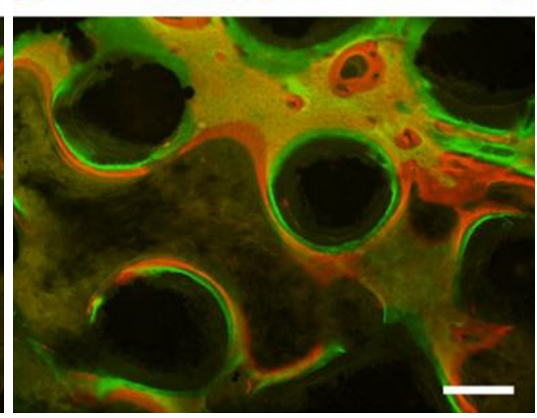

Ti-MBG

Figure 7 The representative merged images of fluorescent double labeling of calcein (green color) and xylenol orange (red color), showing bone formation in the three groups of the porous scaffolds at week 3,6 , and 9 (white scale bar $=200 \mu \mathrm{m}$ ).

and osteoconductivity, the inherent fragility of MBG is a major limitation for its clinical application, especially at load-bearing sites. Coating additively manufactured lattice structures with MBG simultaneously resolves the challenges associated with the fragility of MBG and the limited osteoconductivity of bare metal scaffolds, leading to a marked improvement in the osteointegration of the implants in the host bony tissue. In a previous study, we applied BG and MBG coatings onto the strut surfaces of Ti-6Al-4V scaffolds by the means of spin coating followed by a heat treatment (23), which resulted in the enhanced attachment of hBMSCs on the MBG-coated Ti-6Al$4 \mathrm{~V}$ scaffolds (23). In the present in vivo study, we found that at the early stage after implantation, the Ti-6Al-4V scaffolds coated with BG and MBG exhibited higher bone regeneration capabilities in the central part of the scaffolds, as compared to the bare Ti-6Al-4V scaffolds. At week 6, the quantitative analyses of BV/TV, Tb.Th, Tb.N, and Tb.Sp showed that the bone regeneration capability of the TiMBG group was significantly higher than that of the Ti-BG group $(\mathrm{P}<0.05, \mathrm{n}=4)$. From the MAR values (Figure 8$)$ and the histological images (Figure 9), we found that the most active stage of bone regeneration in the central part of the scaffold was the first 6 weeks after implantation. The good bone regeneration capacity is considered to be related to the microstructure present on the strut surfaces of the scaffolds. In a previous in vitro study (23), we observed that both BG and MBG coatings could release silicon and calcium ions to the Tris-HCl buffer. The ion release ability of the MBG coating was significantly higher than that of the BG coating. 


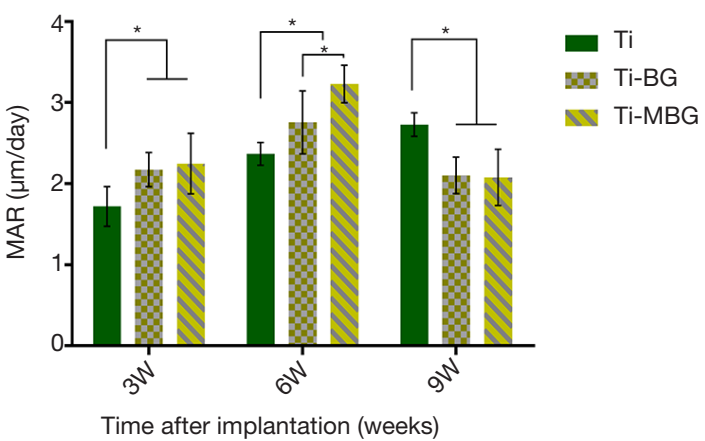

Figure 8 The mineral apposition rates (MAR = distance of fluorescence double labeling/time) of the three groups at week 3, week 6 , and week 9 ( ${ }^{*}$ indicates $\mathrm{P}<0.05$ ).

Given the fact the release of silicon and calcium ions are known to stimulate the regeneration of de novo bone $(43,44)$, the excellent osteoconduction of the MBG-coated scaffolds in bone defect healing could be attributed to the increased release of silicon and calcium ions. Moreover, the presence of a nano-mesoporous structure on the MBG surface could play an important role in this regard. Because of its high specific surface area, MBG can release silicon and calcium ions at a sustained high rate in a biological buffer solution. The ion release and ion-exchange phenomena upon contact with biological fluids may lead to fast apatite formation on the surface of MBG $(34,45)$. As proven in a previous study, nanoscale surfaces may adsorb significantly increased amounts of absorbed protein molecules (particularly positive-charged proteins) (46). The protein adsorption may further mediate cell attachment (47).

The observations made regarding bone regeneration around the periphery of the porous scaffolds were quite similar to those of the central part of the implants. However, at week 9 after implantation, an interesting phenomenon was observed in the analysis of the Tb.N value of each of the groups. The trabecular number in the peripheral part of the MBG-coated porous scaffolds was still on the rise till week 9, while the Tb.N values of the TiBG and Ti groups declined at week 9. Indeed, the presence of blood supply might have enabled a more continuous and stable bone regeneration process at the periphery of the porous scaffolds, as compared to their inner space. This phenomenon suggests that the osteoconduction capability of a porous scaffold depends not only on the type of the surface coating, but potentially also on the location of the coating, the geometrical design of the scaffolds, and the implantation site. The potential effects of the geometrical design of the scaffolds as well as the implantation site should be further investigated in future studies. In the design of the coating protocol, different coatings could be applied to the different parts of the porous scaffold to better guide the process of bone tissue regeneration. For example, the MBG coating may be applied to the struts in the central part of the porous scaffolds, while the BG coating to the struts on the outer surface. To increase the space for new bone formation and vascularization, one may consider a further increase in the porosity of porous scaffolds, a reduction in coating thickness, and an increase in coating roughness.

Bone regeneration is a complex process. The mechanical stabilization of the defect and an adequate host response are both critical for ensuring successful bone formation (48). Furthermore, the re-establishment of an adequate blood vessel system is of great importance for the success of bone regeneration $(49,50)$, particularly when the bony defects are large enough to render diffusion alone insufficient for the oxygenation and nutrition of the cells. By delivering oxygen, nutrients, minerals, and osteoprogenitors to the defect site and over large distances, the angiogenic response aids in restoring the damaged bone (51). The vascular formation is controlled by locally released growth factors and cytokines, particularly under trauma conditions. The type and dose of such growth factors and cytokines must be strictly regulated, to properly regulate the activation, proliferation, and differentiation of skeletal progenitor cells. Under optimum conditions, these processes occur simultaneously, leading to complete restoration of the original morphology and the biomechanical properties of the native bone (52). In this study, mature LB and vascular distribution were observed in the central part of the BG- and MBG-coated scaffolds from week 6. A more typical Haversian system and extensive blood vessel system could be observed in the Ti-MBG group at week 9 . These observations suggest that the vascularization ability of the MBG-coated scaffolds was more enhanced than that of the bare-metal Ti-6Al-4V scaffolds or that of the BG-coated scaffolds. Once more, the release of silicon and calcium ions and the presence of a nano-mesoporous structure of the MBG surface may have played an important role in the commencement and progress of angiogenesis during the bone defect healing process.

The results obtained from the present in vivo study on 3D printed Ti-6Al-4V scaffolds with BG and MBG surface modifications could offer new possibilities for rendering bioactivity to $3 \mathrm{D}$ printed Ti-6Al-4V scaffolds, thereby 


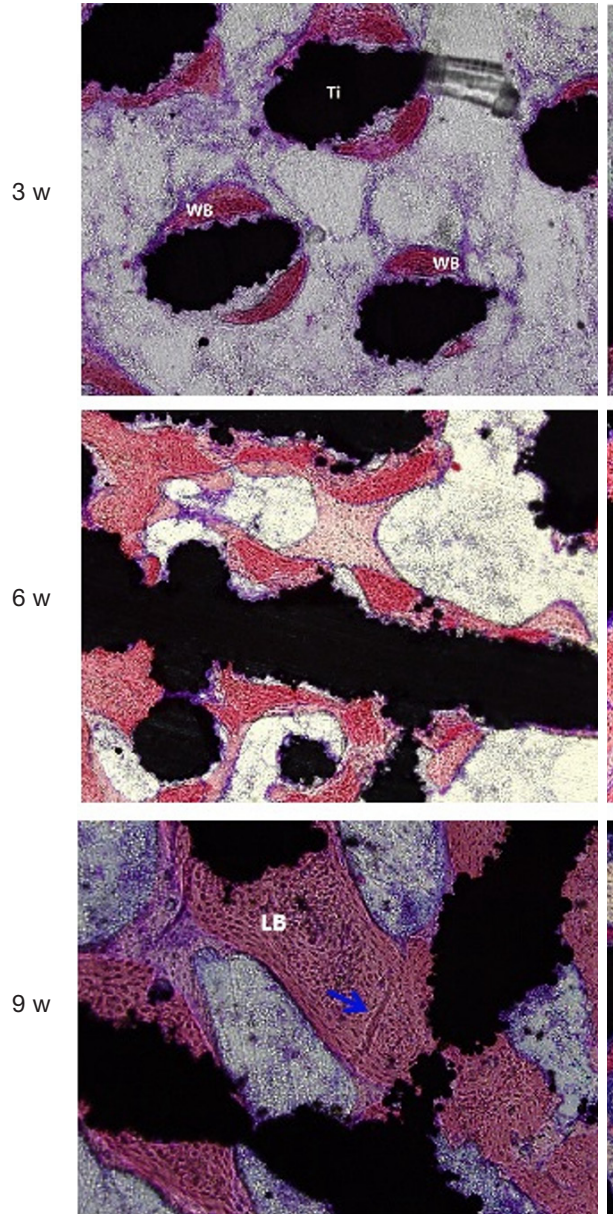

$\mathrm{Ti}$
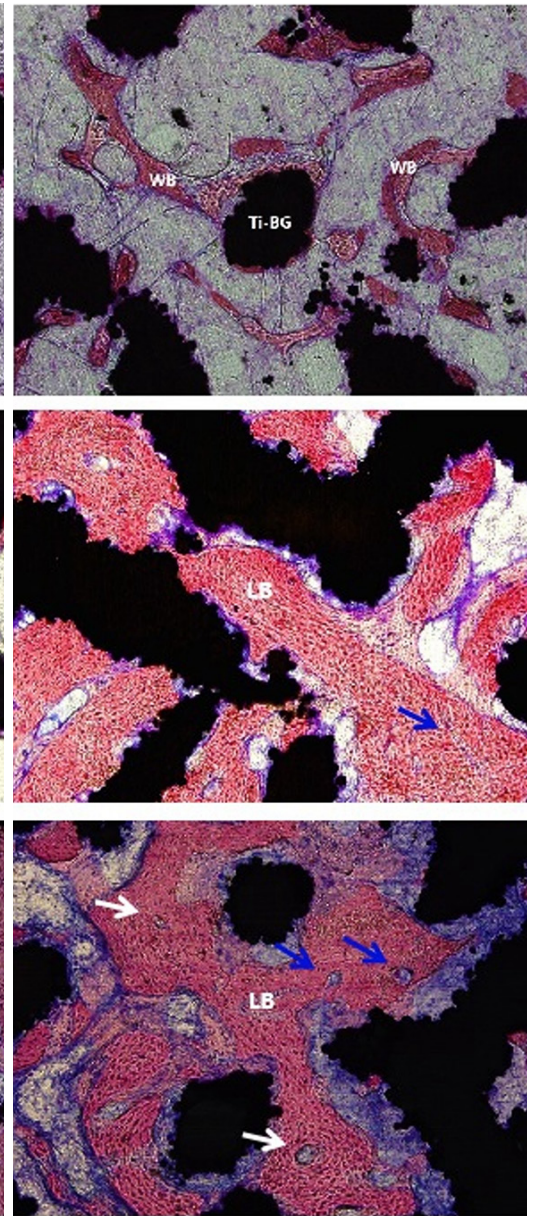

Ti-BG
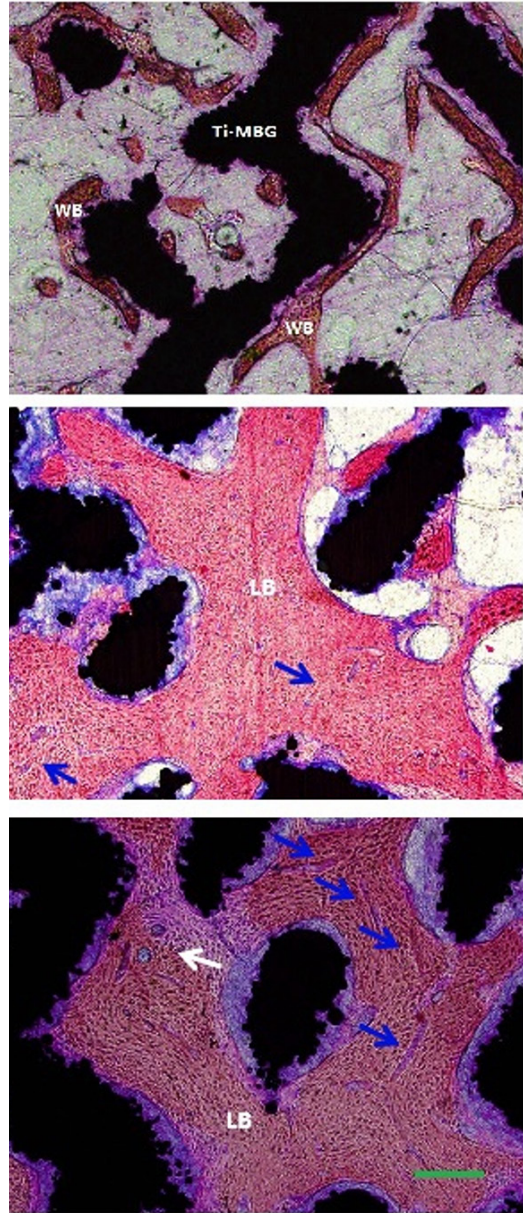

Ti-MBG

Figure 9 The representative histological images of non-decalcified sections obtained using methylene blue acid fuchsin staining. The blue arrows indicate neovascularization, while the white arrows indicate the Haversian system. WB, woven bone; LB, lamellar bone (green scale bar $=200 \mu \mathrm{m})$.

improving their therapeutic efficacy in the treatment of large bony defects such as human maxilla, mandible and non-weight bearing parts of limbs. Further research needs to be directed at understanding the coating-strut adhesion under physiological loads, while the BG and MBG coatings biodegrade overtime during the bone healing phase. Besides, the osteogenesis capacity of porous scaffolds should be evaluated in critical size segmental defect models of loadbearing long bones (e.g., femur, tibia, and radius). Such types of in vivo models would also allow for biomechanical testing, which could be used to evaluate the interface strength of the bone-implant complex and to establish whether or not the process of bone tissue regeneration has progressed far enough to have restored the functional capacity of the long bone in terms of its load-bearing capacity. The degree of restoration of the original anatomy is the other readout of such an experiment.

\section{Acknowledgments}

Funding: We acknowledge the financial support from the National Key Research and Development Program of China (No. 2017YFB1104101), Natural Science Foundation of China (No. 81671830), and Science and Technology Commission of Shanghai Municipality (No. 19441902300).

\section{Footnote}

Reporting Checklist: The authors have completed the ARRIVE reporting checklist. Available at http://dx.doi. 
org/10.21037/atm-20-3829

Data Sharing Statement: Available at http://dx.doi. org/10.21037/atm-20-3829

Peer Review File: Available at http://dx.doi.org/10.21037/ atm-20-3829

Conflicts of Interest: All authors have completed the ICMJE uniform disclosure form (available at http://dx.doi. org/10.21037/atm-20-3829). The authors have no conflicts of interest to declare.

Ethical Statement: The authors are accountable for all aspects of the work in ensuring that questions related to the accuracy or integrity of any part of the work are appropriately investigated and resolved. The study was approved by the Animal Ethics Committee of China Medical University (No. K2016014). The general guidelines on the use of animals in research have complied with all these experiments.

Open Access Statement: This is an Open Access article distributed in accordance with the Creative Commons Attribution-NonCommercial-NoDerivs 4.0 International License (CC BY-NC-ND 4.0), which permits the noncommercial replication and distribution of the article with the strict proviso that no changes or edits are made and the original work is properly cited (including links to both the formal publication through the relevant DOI and the license). See: https://creativecommons.org/licenses/by-nc-nd/4.0/.

\section{References}

1. García-Gareta E, Coathup MJ, Blunn GW. Osteoinduction of bone grafting materials for bone repair and regeneration. Bone 2015;81:112-21.

2. Cuthbert RJ, Churchman SM, Tan HB, et al. Induced periosteum a complex cellular scaffold for the treatment of large bone defects. Bone 2013;57:484-92.

3. Zhang J, Liu W, Schnitzler V, et al. Calcium phosphate cements for bone substitution: Chemistry, handling and mechanical properties. Acta Biomater 2014;10:1035-49.

4. Oryan A, Alidadi S, Moshiri A, et al. Bone regenerative medicine: classic options, novel strategies, and future directions. J Orthop Surg Res 2014;9:18.

5. Tsai $\mathrm{CH}$, Hung $\mathrm{CH}$, Kuo CN, et al. Improved bioactivity of $3 \mathrm{D}$ p rinted porous titanium alloy scaffold with chitosan/magnesium-calcium silicate composite for orthopedic applications. Materials 2019;12:203.

6. Moore WR, Graves SE, Bain GI. Synthetic bone graft substitutes. ANZ J Surg 2001;71:354-61.

7. Tang $\mathrm{W}, \mathrm{Lin} \mathrm{D}, \mathrm{Yu} \mathrm{Y}$, et al. Bioinspired trimodal macro/ micro/nano-porous scaffolds loading rhBMP-2 for complete regeneration of critical size bone defect. Acta Biomater 2016;32:309-23.

8. Turnbull G, Clarke J, Picard F, et al. 3D bioactive composite scaffolds for bone tissue engineering. Bioact Mater 2017;3:278-314.

9. Zadpoor AA, Bone tissue regeneration: the role of scaffold geometry. Biomater Sci 2015;3:231-45.

10. Wu S, Liu X, Yeung KWK, et al. Biomimetic porous scaffolds for bone tissue engineering. Mater Sci Eng R Rep 2014;80:1-36.

11. Bose S, Roy M, Bandyopadhyay A. Recent advances in bone tissue engineering scaffolds. Trends Biotechnol 2012;30:546-54.

12. O'Keefe RJ, Mao J. Bone tissue engineering and regeneration: from discovery to the clinic-an overview. Tissue Eng. Part B Rev 2011;17:389-92.

13. Henkel J, Woodruff MA, Epari DR, et al. Bone regeneration based on tissue engineering conceptions - A 21st century perspective. Bone Res 2013;1:216-48.

14. Bouet $\mathrm{G}$, Marchat $\mathrm{D}$, Cruel M. et al. In vitro threedimensional bone tissue models: from cells to controlled and dynamic environment. Tissue Eng Part B Rev 2015;21:133-56.

15. Rouwkema J, Rivron NC, van Blitterswijk C. Vascularization in tissue engineering. Trends Biotechnol 2008;26:434-41.

16. Barabaschi GDG, Manoharan V, Li Q, et al, Engineering pre-vascularized scaffolds for bone regeneration. Adv Exp Med Biol 2015;881:79-94.

17. Cheng XY, Li SJ, Murr LE, et al. Compression deformation behavior of Ti-6Al-4V alloy with cellular structures fabricated by electron beam melting. J Mech Behav Biomed Mater 2012;16:153-62.

18. Casalino G, Campanelli SL, Contuzzi N, et al. Experimental investigation and statistical optimisation of the selective laser melting process of a maraging steel. Opt Laser Technol 2015;65:151-8.

19. Gibbs DMR, Vaezi M, Yang S, et al. Hope versus hype: what can additive manufacturing realistically offer trauma and orthopedic surgery? Regen Med 2014;9:535-49.

20. Derby B, Printing and prototyping of tissues and scaffolds. Science 2012;338:921-6. 


\section{Page 14 of 15}

21. Melchels FPW, Domingos MAN, Klein TJ, et al. Additive manufacturing of tissues and organs. Prog Polym Sci 2012;37:1079-104.

22. Hutmacher DW, Cool S. Concepts of scaffold-based tissue engineering--the rationale to use solid free-form fabrication techniques. J Cell Mol Med 2007;11:654-69.

23. Ye X, Leeflang S, Wu C, et al. Mesoporous bioactive glass functionalized 3D Ti-6Al-4V scaffolds with improved surface bioactivity. Materials (Basel) 2017;10:1244.

24. Yin B, Xue B, Wu Z, et al. A novel hybrid 3D-printed titanium scaffold for osteogenesis in a rabbit calvarial defect model. Am J Transl Res 2018;10:474-82.

25. Roseti L, Parisi V, Petretta M, et al. Scaffolds for bone tissue engineering: state of the art and new perspectives. Mater Sci Eng C Mater Biol Appl 2017;78:1246-62.

26. Tang D, Tare RS, Yang L-Y, et al. Biofabrication of bone tissue: approaches, challenges and translation for bone regeneration. Biomaterials 2016;83:363-82.

27. Hench LL, Splinter RJ, Allen WC, et al. Bonding mechanisms at the interface of ceramic prosthetic materials. J Biomed Mater Res 1971;5:117-41.

28. Fernandes JS, Gentile P, Pires RA, et al. Multifunctional bioactive glass and glass-ceramic biomaterials with antibacterial properties for repair and regeneration of bone tissue. Acta Biomater 2017;59:2-11.

29. Brauer DS. Bioactive Glasses-Structure and Properties, Angew Chem Int Ed Engl 2015;54:4160-81.

30. Kaur G. Pandey OP, Singh K, et al. A review of bioactive glasses: Their structure, properties, fabrication and apatite formation. J Biomed Mater Res A 2014;102:254-74.

31. Jones JR. Review of bioactive glass: From Hench to hybrids, Acta Biomater 2013;9:4457-86.

32. Miguez-Pacheco V, Hench LL, Boccaccini AR. Bioactive glasses beyond bone and teeth: Emerging applications in contact with soft tissues. Acta Biomater 2015;13:1-15.

33. Yan XX, Deng HX, Huang XH, et al. Mesoporous bioactive glasses. I. Synthesis and structural characterization, J Non-Cryst Solids 2005;351:3209-17.

34. Yan $X$, Huang $X, Y u C$, et al. The in-vitro bioactivity of mesoporous bioactive glasses. Biomaterials 2006;27:3396-403.

35. Zhang Y, Xia L, Zhai D, et al. Mesoporous bioactive glass nanolayer-functionalized 3D-printed scaffolds for accelerating osteogenesis and angiogenesis. Nanoscale 2015;7:19207-21.

36. Cao H, Feng L, Wu Z, et al. Effect of low-intensity pulsed ultrasound on the biological behavior of osteoblasts on porous titanium alloy scaffolds: An in vitro and in vivo
Zhang et al. In vivo study of surface-treated Ti-6Al-4V scaffolds

study. Mater Sci Eng C Mater Biol Appl 2017;80:7-17.

37. Lewis G, Properties of open-cell porous metals and alloys for orthopedic applications. J Mater Sci Mater Med 2013;24:2293-325.

38. Yazdimamaghani M, Razavi M, Vashaee D, et al. Porous magnesium-based scaffolds for tissue engineering, Mater Sci Eng C Mater Biol Appl 2017;71:1253-66.

39. Almubarak S, Nethercott H, Freeberg M, et al. Tissue engineering strategies for promoting vascularized bone regeneration. Bone 2016;83:197-209.

40. Yin $\mathrm{S}$, Zhang $\mathrm{W}$, Zhang $Z$, et al. Recent advances in scaffold design and material for vascularized tissueengineered bone regeneration. Adv Healthc Mater 2019;8:e1801433.

41. Javadi A, Solouk A, Nazarpak MH, et al. Surface engineering of titanium-based implants using electrospraying and dip coating methods. Mater Sci Eng C Mater Biol Appl 2019;99:620-30.

42. Huynh V, Ngo NK, Golden TD. Surface activation and pretreatments for biocompatible metals and alloys used in biomedical applications. Int J Biomater 2019;2019:3806504.

43. Lee MN, Hwang H-S, Oh S-H, et al. Elevated extracellular calcium ions promote proliferation and migration of mesenchymal stem cells via increasing osteopontin expression. Exp Mol Med 2018;50:1-16.

44. Shi M, Zhou Y, Shao J, et al. Stimulation of osteogenesis and angiogenesis of hBMSCs by delivering Si ions and functional drug from mesoporous silica nanospheres. Acta Biomater 2015;21:178-89.

45. Lammens J, Laumen A, Delport H, et al. The Pentaconcept in skeletal tissue engineering. A combined approach for the repair of bone defects. Acta Orthop Belg 2012;78:569-73.

46. Baino F, Fiorilli S, Vitale-Brovarone C. Bioactive glassbased materials with hierarchical porosity for medical applications: Review of recent advances. Acta Biomater 2016;42:18-32.

47. Kang MS, Lee N-H, Singh RK, et al. Nanocements produced from mesoporous bioactive glass nanoparticles. Biomaterials 2018;162:183-99.

48. Lavenus S, Poxson DJ, Ogievetsky N, et al. Stem cell behavior on tailored porous oxide surface coatings. Biomaterials 2015;55:96-109.

49. Romero-Sánchez LB, Marí-Beffa M, Carrillo P, et al. Copper-containing mesoporous bioactive glass promotes angiogenesis in an in vivo zebrafish model. Acta Biomater 2018;68:272-85. 
50. Baino F, Novajra G, Pacheco VM, et al. Bioactive glasses: Special applications outside the skeletal system. J NonCryst Solids 2016;432:15-30.

51. Stegen S, Gastel N, Carmeliet G. Bringing new life to damaged bone: The importance of angiogenesis in bone repair and regeneration. Bone 2015;70:19-27.

Cite this article as: Zhang G, Zhao P, Lin L, Qin L, Huan Z, Leeflang S, Zadpoor AA, Zhou J, Wu L. Surface-treated 3D printed Ti-6Al-4V scaffolds with enhanced bone regeneration performance: an in vivo study. Ann Transl Med 2021;9(1):39. doi: 10.21037/atm-20-3829
52. Santos MI, Unger RE, Sousa RA, et al. Crosstalk between osteoblasts and endothelial cells co-cultured on a polycaprolactone-starch scaffold and the in vitro development of vascularization. Biomaterials 2009;30:4407-15. 